\title{
José Miguel Alfaro (1940-2013), in memóriam
}

\author{
Rafael Ángel Méndez Alfaro \\ Historiador costarricense. Coordinador del Programa de Estudios Generales de la Universidad Estatal a Distancia (UNED) \\ y profesor asociado de la Universidad de Costa Rica (UCR). Premio Cleto González Víquez de la Academia de Geografía \\ e Historia de Costa Rica (2007). Correo electrónico: ramendez@uned.ac.cr
}

Recibido: Febrero 2014 • Aceptado: Marzo 2014

A inicios del mes de setiembre del 2013, falleció el abogado, político y académico costarricense José Miguel Alfaro, figura destacada del ámbito público local y regional, docente universitario y estudioso formal de los vínculos de nuestro país con sus homólogos de la región centroamericana, razón por la cual fue llamado con toda justicia por los estudiosos de la temática "padre del derecho comunitario en Costa Rica", área donde dejó constancia de una fructífera producción intelectual desde una época temprana de su vida hasta años recientes.

Reconocido por su destacada labor como vicepresidente de la República, durante la administración de Rodrigo Carazo Odio (1978-1982) -gestión donde se desempeñó, de forma adicional, como encargado de la Cartera de Trabajo y Seguridad Social en la primera parte de dicho gobierno y como Ministro de Economía, Industria y Comercio, a partir del 1 de junio de 1980, en una coyuntura particularmente compleja en materia fiscal-, Alfaro se constituyó en el rostro visible que lideró un ministerio estratégico en la toma de decisiones, en una época convulsa para la economía costarricense.

Asimismo, actuó como ministro encargado de Asuntos de Integración ante los organismos del Mercado Común Centroamericano, fue director del Banco Central y coordinador de la política de negociación de la deuda externa. Semejantes responsabilidades en áreas de acción de distinta naturaleza lo colocan como una figura fundamental en la administración Carazo Odio. En este punto, la percepción de los especialistas, independientemente de su origen y posiciones, coinciden en la valiosa contribución que su gestión brindó a Costa Rica en ese lapso.

Académico por excelencia, se graduó como abogado en la Universidad de Costa Rica (UCR), centro de estudios en el que fue profesor en la Escuela de Derecho desde mediados de la década de 1960. Dentro de su trayectoria se destacó como docente de los cursos Introducción al Estudio del Derecho y Teoría del Estado. Además, cabe subrayar que fue la figura encargada de fundar la Cátedra de Derecho Comunitario Centroamericano, temática que estudió con detenimiento y en la cual fue considerado un especialista por académicos y políticos de toda Centroamérica, dejando constancia de ello en sus escritos y conferencias en todos los países del istmo.

Consejal externo en el Consejo Universitario de la Universidad Estatal a Distancia (UNED), donde promovió y participó en fértiles discusiones 
en torno al tema país; profesor invitado en diversas universidades extranjeras y en la Universidad para la Paz, que él mismo ayudó a levantar siendo vicepresidente de la República en el período ya señalado. José Miguel Alfaro fue un activo intelectual a lo largo de su carrera. Moderador de mesas en los campus universitarios donde se analizaba el contexto contemporáneo, expositor, conferencista, presentador de libros y participante en seminarios, desarrolló una febril actividad académica de manera permanente, razón por la cual fue una figura constantemente citada por los estudiosos de la realidad centroamericana.

La presente reseña es solo una aproximación que procura recuperar parte de la vida y obra de Alfaro. En ese sentido, tal esfuerzo se enmarca dentro de la iniciativa del Consejo Universitario de la Universidad Estatal a Distancia, que por acuerdo del 10 de setiembre del 2013 (CU-2013511), determinó solicitar a la Cátedra El País Que Necesitamos una reseña de la labor y trayectoria del señor Alfaro Rodríguez. Bajo el liderazgo del doctor Humberto Aguilar Arroyo, director de la Escuela de Ciencias Sociales y Humanidades -quien asumió de forma directa el mandato del Consejo Universitario, y con el aporte del Programa de Estudios Generales-, se presenta este in memóriam a José Miguel Alfaro (1940-2013).

\section{El padre del derecho comunitario en Costa Rica}

El III Seminario sobre Integración Centroamericana y Derecho Comunitario, convocado para setiembre del 2013 y auspiciado, entre otros, por la Universidad Nacional (UNA), la Universidad de Costa Rica, el Colegio de Abogados y Abogadas de Costa Rica, la Corte Suprema de Justicia, el Instituto de Estudio e Investigación Jurídica (INEJ, Nicaragua) y el Sistema de la Integración Centroamericana (SICA), tenía en principio entre sus expositores previstos a José Miguel Alfaro en la mesa número uno, que presentaba como tema principal "Crecimiento económico y cohesión social". La infausta partida del reconocido estudioso de la realidad centroamericana trajo como secuela directa que el seminario se dedicara a su memoria, como reconocimiento a su constante labor en la promoción del derecho comunitario.

Sin embargo, más allá de ese justo homenaje, brindado por los organizadores del evento académico, la participación prevista de Alfaro es una evidencia del nivel de compromiso con la causa centroamericana y de una actividad intelectual constante, aspecto que caracterizó toda su trayectoria. Sus frecuentes participaciones durante los últimos años en mesas de discusión sobre la situación regional, participando en el debate y análisis de temáticas tan trascendentes como el golpe de Estado en la República de Honduras, las bases conceptuales de la democracia en la sociedad contemporánea y el papel de Costa Rica en los procesos de integración en el istmo, lo convirtieron en un referente necesario por considerar en las agendas de análisis de quienes promueven el estudio de la realidad social de nuestros países.

Estudioso incansable, integró el Comité organizador y fundador de la Maestría en Derecho Comunitario y Derechos Humanos. No solo fue gestor de esta iniciativa, sino que cursó dicho programa, constituyéndose en egresado de la primera generación (2009-2011), con su investigación "El orden sistémico del SICA". Los elementos aquí mencionados colocan a Alfaro como un estudioso de primer orden en esta materia. De hecho, habría que apuntar en esta misma dirección que fue miembro de la Comisión ad hoc para el replanteamiento del Sistema de la Integración Centroamericana en el lapso 2004-2007, y coordinó la Comisión Centroamericana de Juristas de la Integración en el período 1991-1995. Su reputación como especialista en derecho comunitario le valió múltiples reconocimientos, tanto en Costa Rica como en países hermanos de la región.

En junio del 2013, fue designado por la Secretaría por la Secretaría General del Sistema de Integración Centroamericana (SICA) como jurista e integracionista notable, por haber formado parte del grupo de expertos que elaboraron el Protocolo de Tegucigalpa (81991), a la Carta de la Organización de Estados Centroamericanos (ODECA). A partir de esa fecha, en la sede del 
SICA, en San Salvador, su nombre ocupa el Salón José Miguel Alfaro Rodríguez. De hecho, sus contribuciones al SICA fueron desde el plano de la asesoría hasta la organización propiamente dicha de eventos claves en la toma de decisiones de este organismo ístmico (Alfaro, 2013: 1-18).

Su condición de hombre propulsor de la integración regional lo llevó desde una época temprana a ser consultor externo de instancias como el Ministerio de Relaciones Exteriores de Costa Rica, el Ministerio de Comercio Exterior (COMEX), la Secretaría General de la Integración Centroamericana (SG-SICA), la Secretaría General del Sistema de Integración Económica (SIECA), la Secretaría del Consejo Agropecuario Centroamericano (SG-CAC), la Secretaría de la Coordinación Educativa y Cultural Centroamericana (SG-CECC), la Secretaría del Consejo Monetario Centroamericano (SG-CMCA), el Instituto de Nutrición de Centroamérica y Panamá (INCAP) y el Instituto Centroamericano de Administración Pública (Cuevas y otros, 2004).

En esa misma dirección, Alfaro asesoró en distintos momentos instancias de la región como el Consejo Universitario Centroamericano (CSUCA), la Secretaría del Comité Regional de Acción Social (SG-CRAS), el Banco Centroamericano de Integración Económica (BCIE), el Centro para la Prevención de Desastres Naturales en América Central (CEPREDENAC), el Comité Coordinador Regional de Instituciones de Agua Potable y Saneamiento de Centroamérica, Panamá y República Dominicana (CAPRE), la Corporación Centroamericana de Servicios de Navegación Aérea (COCESNA), la Federación de Entidades Privadas de Centroamérica y Panamá (FEDEPRICAP), la Federación de Cámaras de Industrias de Centroamérica (FECAICA), la Corte Centroamericana de Justicia (CCJ) y el Comité Coordinador de Empresarios de la Microempresa de la Región Central de América (Cuevas y otros, 2004).

Enrique Castillo, actual ministro de Relaciones Exteriores de Costa Rica, calificó a José Miguel Alfaro como un ilustre jurista de la causa centroamericana y destacó su contribución, en ese sentido, como notable. Gioconda Ubeda, vicecanciller de la República, no dudó en reconocer el don de gentes, la abierta disposición de colaboración, la sensibilidad social, el conocimiento, la entrega y el compromiso con la integración centroamericana que caracterizó la vida de José Miguel Alfaro (<www.rree.go.cr>). De hecho, por largos años Alfaro fue un asesor regular de la Cancillería en asuntos que guardaban estrecha relación con temas centroamericanos.

Alfaro no solo fue un activo promotor de la integración, sino también un profundo estudioso de este proceso. Su obra escrita es la mejor evidencia de ello:

Esa Costa Rica en crisis, trata de encontrar su espacio en la región, ayudando a fortalecer los procesos de pacificación, democratización y mejoramiento de la calidad de vida y uniendo sus esfuerzos para que la región, como un todo, pueda vencer los antivalores que la mantiene violenta en un mar de exclusión (Alfaro, 2002: 91).

La cita previa deja constancia del interés primordial que siempre privó en la visión de José Miguel Alfaro. Pensar Costa Rica incorporada a la región centroamericana, en un esfuerzo mancomunado por el desarrollo del istmo, donde se potencian las condiciones y fortalezas del país. Sobre aspectos como democracia y paz, Costa Rica tiene mucho que aportar y desde ese punto de vista, el pensamiento de Alfaro procura recuperar aquellos elementos históricos que han caracterizado la evolución de nuestro país.

Sobre el ser humano, Alfaro dejó destacadas reflexiones sobre su situación y perspectivas en una región como Centroamérica, tal como se aprecia a continuación:

El ser humano es autor, centro y fin de todo el proceso económico social, nos recordó el Papa Juan XXIII.

No es el estado, ni la economía, ni el poder, ni la dictadura, ni la oligarquía, ni la raza, ni la religión, ni el partido político. Es el ser humano quien crea, centra y es la razón del ser del proceso económico social.

Hecho a imagen y semejanza de Dios, capaz de conocer y discernir, de aprender y aprehender, capaz de decidir y escoger, capaz de amar y crear. 
Ese ser humano al que las estructuras de poder han querido mantener reducido a simple y descarnado factor de producción, de consumo, de riqueza para los demás.

Ser que sueña, lucha y se inmola para alcanzar los ideales de paz, justicia y libertad.

Ser que anhela un futuro mejor para sus hijos, que los quiere sanos, educados, cultos, con un nivel de vida digno (Alfaro, 2002: 91-92).

Desde la perspectiva del ser humano, encontramos el fértil pensamiento de José Miguel Alfaro que plantea como principal reto del istmo el rescate e incorporación a la construcción y los beneficios de la sociedad organizada de nuestros excluidos y marginados. Es decir, la integración -más que procesos de naturaleza económica y política- debe consistir en iniciativas tendientes a disminuir la brecha social, a incorporar a la sociedad en plenitud de condiciones a los sectores menos favorecidos, a aquellos a los cuales la desigual distribución de la riqueza los ubica en una condición de fragilidad y deterioro.

Desde el punto de vista del proceso de la integración centroamericana, Alfaro ubica al ser humano inserto en un conjunto de preocupaciones absolutamente válidas en relación con las condiciones que deben prevalecer para hacer realidad su realización. Al respecto plantea lo siguiente:

El ser humano anhela la oportunidad de realizarse plenamente como persona, en familia y en comunidad. Aspira a reafirmar su autoestima, a ser partícipe en la construcción y en los beneficios de la sociedad organizada. Ve el proceso de desarrollo como un proyecto de liberación integral (Alfaro, 2002: 95 ).

En este sentido, la integración del istmo debe ser capaz de promover espacios que lleven al ser humano social, solidario y comunitario (ideal predominante en el pensamiento de Alfaro), a la realización digna de la vida humana. El pensamiento de Alfaro, que evidencia un fuerte influjo de la doctrina social de la Iglesia católica, busca conciliar procesos que se asocian con las estructuras productivas y los ámbitos de poder en la toma de decisiones, con la aspiración de la realización del ser humano. Esto es una constante que se refleja en su obra y vida.

La revisión de parte de su producción intelectual, ligada a su gran pasión por el estudio de la integración centroamericana, revela un interés constante por los retos que debe enfrentar el istmo y las acciones por tomar para asumir el compromiso de orientar el desarrollo de la región. En su trabajo La integración como instrumento de desarrollo: sus perspectivas y desafios para Centro América. Contribuciones de un seminario regional del INCEP (Alfaro, 1996) se presenta precisamente el planteamiento central de su obra, y es que en gran medida, las alternativas del desarrollo para las naciones de la región pasan, de forma primordial, por los procesos de integración que se puedan ejecutar, por los acuerdos a los que puedan llegar los países involucrados y por la voluntad política existente para conciliar intereses que superen las perspectivas de carácter nacional y se incrusten en propuestas de carácter regional.

$\mathrm{Su}$ investigación -escrita junto con Carlos Manuel Echeverría- Procesos de integración centroamericana como región de paz, libertad, democracia y desarrollo (Alfaro y Echeverría, 1996), publicada bajo el patrocinio de la Universidad para la Paz, retoma la importancia estratégica que tiene la integración regional como mecanismo efectivo capaz de transformar la realidad de las naciones centroamericanas en un escenario propicio para el desarrollo con sentido humano. Es decir, un desarrollo que tenga impacto en la situación particular de las personas.

Junto a Rafael Cuevas Molina, Jaime Delgado y Carmen Camacho produjo la obra Integración en Centroamérica: cultural, social, politica y económica (Cuevas y otros, 2004), obra que reúne el aporte de un conjunto de especialistas en la materia y propone un conjunto de reflexiones sobre el alcance de la integración en sus distintas manifestaciones. Esta obra es una evidencia adicional del compromiso que en vida y obra llevó a cabo José Miguel Alfaro en un tema que representó una pasión de primer orden en su itinerario de vida. 


\section{Un hombre de trayectoria}

Tuve la suerte - en mi caso-de ser estudiante cuando él era profesor de la Facultad de Derecho, en la cátedra de Derecho comunitario. Cuando estaba empezando el tema del mercado común centroamericano, José Miguel Alfaro fue de los visionarios que vieron esa oportunidad y que trabajaron y que crearon todo un sistema jurídico para darle pie a lo que fue el mercado común centroamericano.

Me tocó, en 1972, estar junto a él en la fundación del Partido Renovación Democrática, en el Colegio Los Ángeles, el 15 de setiembre, y José Miguel Alfaro ahí estaba presente. En 1978 don José Miguel Alfaro, como vicepresidente, todavía en la Casa Presidencial vieja, trabajábamos, y yo era asistente de don Rodrigo Carazo, y don José Miguel era, yo diría, que de las personas de más confianza, a quien don Rodrigo Carazo confiaba la mayoría de las situaciones, por su lealtad, por su transparencia, por su valentía (Asamblea Legislativa, Sesión Plenaria $n{ }^{\circ} 58$, del 2 de setiembre del 2013: 11-12).

La cita previa forma parte de la participación que Luis Fishmann tuvo en la Asamblea Legislativa, en la sesión plenaria n. ${ }^{\circ} 58$ del 2 de setiembre del 2013, cuando por motivo de la partida de José Miguel Alfaro se dedicó un minuto de silencio en su honor. En ella se encuentran manifiestos dos aspectos medulares que es preciso destacar; en primer lugar, la faceta de profesor universitario asociado con intereses integracionistas desde una época muy temprana, y en segunda instancia, su papel político en la construcción de la oposición al Partido Liberación Nacional durante la década de 1970, esfuerzo que se materializó con el arribo al poder, en 1978, de la coalición Unidad, bajo el liderazgo de Rodrigo Carazo Odio.

La vida de Alfaro en la función pública no fue prolongada, ciertamente. Fue muy intensa durante la administración Carazo Odio, pues adicional a su ejercicio como vicepresidente de la República, tuvo bajo su dirección los ministerios de Trabajo, de Seguridad Social y el de Economía, Industria y Comercio. De paso, asumió la representación del país en los asuntos internacionales que guardaban estrecha relación con los procesos de integración centroamericana, área en la que venía forjando una ruta clara y precisa desde antes de su arribo al Poder Ejecutivo.

La participación (en la misma sesión plenaria anotada líneas atrás) de la diputada Alicia Fournier Vargas muestra impresiones adicionales sobre la trayectoria de José Miguel Alfaro en Costa Rica:

Siempre de la mano de los trabajadores. Me acuerdo, hace cerca de ocho años, desfilando con él hacia Cartago, con todos los productores arroceros de ese momento. Él era el primero que estaba siempre en esas luchas justas de los sectores sociales de nuestro país.

Pero también hay que destacar al jurista centromericanista; al hombre que ayudó a la integración centroamericana, al Sistema de Integración Centroamericana, y hasta hace unos pocos dias - podríamos hablar-siempre fue asesor de nuestra Cancillería en este tema, y siempre prestó, con toda la mayor voluntad, sus servicios y sus conocimientos a nuestro país en este campo, sobre todo, centroamericano.

Yo creo que hay que reconocer que hoy perdimos a un gran hombre, a un gran costarricense, muy sensible, que representa, de veras, a un sector que no debemos olvidar, sobre todo los politicos. A un hombre decente, un hombre honrado y un hombre de veras que prestó sus servicios a la patria (Asamblea Legislativa, Sesión Plenaria ${ }^{\circ}{ }^{\circ}$ 58, 2 de setiembre del 2013: 11).

La cita muestra la imagen de un hombre constantemente preocupado por el tema de la integración regional, estudioso de la situación costarricense en materia jurídica en sus vínculos con los demás países de la región, asesor constante en esa materia de la Cancillería de la República, militante de las causas sociales, honesto y servidor del país.

La recuperación de la intervención del diputado Edgardo Araya Pineda en la sesión legislativa anotada resulta un buen ejemplo de la impresión que en el mundo de la política costarricense se tenía de la figura de José Miguel Alfaro y de la calidad de persona que era:

También como jefe de fracción y como diputado quiero unirme a este minuto de silencio del ex vicepresidente de la República, don José Miguel Alfaro, 
persona a quien conocí en la Municipalidad de San Ramón siendo presidente municipal allá por 1998.

Don José Miguel estuvo muy cercano a nuestro cantón con temas de conservación de medio ambiente, junto con doña Estrella de Carazo; muchos... por mucho tiempo estuvimos allá en Villa Blanca analizando temas de medio ambiente y de, incluso, energías limpias. Se volvió un asesor ad honórem de la Municipalidad de San Ramón hasta hace muy poco tiempo y hace creo que menos de un año estuvimos con él coordinando temas también relacionados con estos dos aspectos; mejoras de asuntos comunales también por allá (Asamblea Legislativa, Sesión Plenaria ${ }^{\circ}{ }^{\circ}$ 58, 2 de setiembre del 2013: 12-13).

Junto a su condición de asesor de la Municipalidad de San Ramón -destacada por el diputado Edgardo Araya-, es justo enfatizar que José Miguel Alfaro fue consultor y asesor de la Alcaldía de Goicoechea y de Curridabat, así como de la Unión Cantonal de Asociaciones de Desarrollo Cantonal de San Ramón; además se desempeñó como magistrado suplente de la Sala Tercera en el lapso 1991-1995, y de la Sala Constitucional de la Corte Suprema de Justicia en el período 19972005, aspectos que en su conjunto muestran solo de forma parcial los diversos escenarios en los cuales se desenvolvió (Asamblea Legislativa, Sesión Plenaria, . $^{\circ}$ 58, 2 de setiembre del 2013).

$\mathrm{Su}$ faceta de magistrado, tanto de la Sala Tercera como de la Sala Constitucional, lo colocan en una situación especial de la historia jurídica costarricense, pues no solo se destacó en el derecho comunitario, sino que su trayectoria lo ubicó en escenarios judiciales locales de primer orden.

Las palabras de Alfaro - a propósito de la figura del expresidente costarricense, Rodrigo Carazo Odio- lo muestran como un ser humano cálido, generoso y leal, aspectos en los que propios y extraños ofrecen criterios similares:

Rodrigo convocó a los ciudadanos para que con él al frente: - "yo doy la cara, yo pongo el pecho y ustedes vienes detrás"- construyéramos una Costa Rica "mejor para todos", tomando el camino del "progreso con dignidad". Condujo al país por esa senda enfrentando la crisis del café, la crisis del petróleo, la crisis de la deuda, la década perdida para
América Latina y la década de sangre para Centroamérica, la sorda oposición de intereses creados.

Gobernó para el bien común de los costarricenses, más de catorce mil obras públicas (más de cuatro mil de ellas con participación comunal). Llevó los programas de salud rural a todos los cantones rurales. Extendió la cobertura de electrificación, telefonía y seguro social. Creó las ferias del agricultor y subsidió el consumo básico por medio de los estancos del Consejo Nacional de Producción. Se enfrentó a las políticas del Fondo Monetario Internacional, a Reagan por un lado y a los rusos por otro, luchando por la paz de la región. Fue el Presidente de todos los costarricenses. Su administración trabajó con líderes comunales y con líderes de todos los colores políticos, sin jamás privilegiar o discriminar a nadie por su credo politico.

Fue, hasta el último aliento, el ex Presidente digno y comprometido. Presente siempre para dar el consejo sabio y oportuno, la directriz certera a todos. Impresionante testimonio lo dan sus agendas, en la que consta cada visita, conferencia, reunión o clase (Zeledón, 2011: 85).

Las palabras precedentes honran la figura de José Miguel Alfaro, en tanto dejan ver la lealtad que siempre sintió por él Carazo, a quien acompañó de cerca en una de las coyunturas más complejas vividas en Costa Rica durante los últimos 40 años de nuestra historia.

\section{Desde el Consejo Universitario}

La autonomía universitaria es un fuero, un privilegio que la Constitución Política da originalmente a la Universidad de Costa Rica y posteriormente a las universidades públicas, para que sea la Asamblea Universitaria Representativa la que legisle en materia universitaria, esto es muy importante, porque la Asamblea Legislativa representa al pueblo y ejerce a nombre del pueblo la facultad de legislar y se consideró por el Constituyente del 49 tan importante la autonomía de la Universidad, que hizo una excepción a esa representación del pueblo y permitió que un órgano particular, es decir, la Asamblea de una Universidad, legisle en materia universitaria, considero que es fundamental que lo entendamos, porque incluso impone una gran responsabilidad 
sobre la Asamblea Universitaria Representativa, que es mucho más que una autoridad que fija pautas para una universidad, sino que está ejerciendo la potestad de legislar, el pueblo normalmente delega en la Asamblea Legislativa, pero que por excepción, a las universidades se les ha dado este fuero, por esta razón es muy importante la salvaguarda de esto (Asamblea Representativa Universitaria, Acta No 081-2010, 5 de marzo de 2010).

Esta cita, extraída de una participación de José Miguel Alfaro en el campus de la Universidad Estatal a Distancia, a propósito del tema de la autonomía universitaria, refleja no solo la claridad conceptual que tenía en esta materia, sino deja ver la importancia de preservar un recurso de esta naturaleza. La autonomía es hoy un elemento fundamental del ser de la universidad en Costa Rica y reflexionar sobre sus alcances parece una obligación de primer orden para toda la comunidad universitaria. De ahí la trascendencia de poner sobre la mesa un tema de semejante alcance.

Habría que señalar que durante su participación como representante externo en el Consejo Universitario de la UNED (2007-2012), Alfaro tuvo como una preocupación central la situación del país en distintas materias. Cuestiones relacionadas con la defensa del Instituto Costarricense de Electricidad, del Poder Judicial y de la institucionalidad fueron asuntos de constante referencia.

La Universidad también reconoce el interés permanente que tuvo sobre los centros universitarios: su funcionamiento, la urgencia de fortalecer las sedes en materia de infraestructura, el equipamiento de las instalaciones y la necesidad de crear las condiciones adecuadas de tipo laboral para el óptimo desempeño de los funcionarios. Por ello, no es de extrañar que se le viera participando activamente en sesiones de trabajo en que se discutían asuntos que guardan estrecha relación con la forma en que se lleva a cabo el trabajo en los centros universitarios, o que se le observara formando parte, como un integrante más de la comunidad unediana, de actos protocolarios donde se inauguraban instalaciones, las cuales hacen más propicio el trabajo en dichos centros.
De igual manera, su nombre aparece asociado de forma directa con las gestiones y discusiones que dieron como resultado la creación de la Vicerrectoría de Investigación en la UNED. Esta, que era una necesidad institucional de primer orden, se implementó como resultado de fértiles discusiones generadas en el interior del Consejo Universitario. La creación de la Vicerrectoría de Investigación se estableció en los siguientes términos:

Solicitar a la Rectoría que, a más tardar el 30 de enero del 2008, presente al Consejo Universitario, para su conocimiento, un informe que contemple la organización interna de la Vicerrectoría de Investigación, así como las acciones estratégicas y el plan operativo anual del 2008 para la puesta en marcha de esta Vicerrectoría, de conformidad con la aprobación de su creación, dada por la Asamblea Universitaria Representativa el 6 de diciembre del 2007 (Consejo Universitario, sesión 1897-2007, correspondiente al 7 de diciembre del 2007).

Dentro de las actas de trabajo del Consejo Universitario se destaca el involucramiento de José Miguel Alfaro dentro de la discusión en torno al proyecto de ley sobre la creación de la Comisión Nacional del Rescate de Valores, instancia de la que formó parte. De hecho, la recuperación de los valores costarricenses fue una constante en el pensamiento y obra de Alfaro.

Lo anterior se vio reflejado de forma constante en la participación de Alfaro en un programa radial a su cargo en la emisora Radio María. Bajo el nombre Somos Su Pueblo, Alfaro presentaba semanalmente un programa con invitados especiales, donde analizaba aspectos medulares de la realidad nacional, vistos a través de la óptica cristiana. Es visible un interés, en estos programas hoy disponibles en la web, por el rescate de valores y por la reflexión acerca de la vida cotidiana de los costarricenses.

En el último de los programas disponibles en el sitio web de Radio María, en agosto del 2013 y con motivo de un programa previo con la historiadora Elizabeth Fonseca, José Miguel Alfaro se permite reflexionar sobre la forma en que principios fundamentales del cristianismo son también 
principios fundamentales de la nacionalidad costarricense. Allí llegó a plantear con claridad que el principio fundamental de convivencia que tiene el cristianismo es la ley del amor (<www.radiomaria.cr $>$ ).

Bajo este precepto, Alfaro sostiene que precisamente quienes organizaron las bases del Estado costarricense a partir de 1821, lo hicieron guiados en principios de armonía, concordia y solidaridad, elementos que son una evidencia de las formas en que se manifiesta el amor. Nunca dudó en señalar que gran parte del recorrido histórico del Estado y la sociedad costarricense estuvo entrañablemente ligado al desarrollo de principios de naturaleza cristiana.

De entre sus múltiples participaciones dentro del Consejo Universitario de la UNED, cabe destacar su propuesta de pronunciamiento de la institución en relación con el golpe de Estado vivido en Honduras, hacia fines de junio del 2009. Su preocupación por la realidad centroamericana lo llevó a presentar ante el seno del Consejo la necesidad de que la Universidad se pronunciara ante un asunto tan delicado como era la ruptura del orden constitucional en el hermano país del istmo. Este tema, que conmovió profundamente a la región latinoamericana, no pasó desapercibido para la aguda visión de Alfaro, de ahí su interés para que la Universidad Estatal a Distancia, como institución promotora de la cultura, no dejara pasar esta oportunidad para hacer manifiesta su posición sobre un asunto en extremo delicado.

En sesión de Consejo Universitario 19872009, correspondiente al 16 julio 2009 (artículo IV, inciso 2), se determinó acoger la propuesta de acuerdo presentada por José Miguel Alfaro (REF. CU-256-2009), sobre la situación vivida por Honduras y se acordó publicar el siguiente pronunciamiento:

\section{CONSIDERANDO QUE:}

1. El Presidente Constitucional de Honduras fue depuesto de su cargo y sacado manu militari de su país.
2. El recurso a la fuerza de las armas constituye una acción violatoria del orden constitucional en Honduras, y de los compromisos básicos del Estado Hondureño con el Sistema de la Integración Centroamericana (SICA), con la Organización de los Estados Americanos (OEA) y la Organización de Naciones Unidas (ONU).

3. El uso de la fuerza militar para interrumpir el funcionamiento normal de las instituciones es incompatible con la democracia y la libertad de los pueblos.

4. El uso de la fuerza militar es la negación del derecho.

5. La situación de violencia institucional que se vive en Honduras, es incompatible con el proceso de transformación de Centroamérica en Región de Paz, Libertad, Democracia y Desarrollo.

6. Corresponde a la comunidad universitaria velar por la salvaguarda de los valores éticos, cívicos y democráticos del pueblo centroamericano.

7. Ante el gravísimo daño que esta acción causa al proceso de democratización en Honduras.

\section{SE ACUERDA:}

1. Condenar el golpe de estado en Honduras.

2. Apoyar el proceso de mediación para lograr la restauración del orden institucional democrático en la República de Honduras.

3. Poner a disposición del Dr. Óscar Arias Sánchez, Presidente Constitucional de Costa Rica, los recursos tecnológicos de la UNED, en lo que puedan servir al proceso de mediación.

4. Hacer votos por una pronta solución por la vía pacífica que permita, a las hermanas y hermanos hondureños, recuperar la normalidad institucional democrática.

ACUERDO FIRME (Consejo Universitario de la Universidad Estatal a Distancia, sesión 19872009, correspondiente al 16 julio 2009). 
Propuestas de esta naturaleza resultan un claro ejemplo de las preocupaciones que en relación con el país y su entorno regional tuvo José Miguel Alfaro a lo largo de su vida y, más aún, revelan mucho de su naturaleza de hombre propositivo, negociador y de principios y valores inquebrantables.

Hoy, la Universidad Estatal a Distancia, en un acto significativo en su vida institucional, como es la conmemoración de la declaración como Institución Benemérita de la Educación y la Cultura de Costa Rica, se complace en dar este sentido y justo homenaje in memóriam a José Miguel Alfaro Rodríguez.

\section{Bibliografía}

Alfaro, José Miguel (1996). La integración como instrumento de desarrollo: sus perspectivas y desafios para Centro América. Contribuciones de un seminario regional del INCEP. Guatemala: Instituto Centroamericano de Estudios Políticos.

Alfaro, José Miguel (2002). Reflexiones sobre Centroamérica. Aspectos jurídicos y políticos de la integración. Heredia: Universidad Nacional.

Alfaro, José Miguel (2013). Una idea-fuerza inicial. Intervención del Lic. José Miguel Alfaro Rodríguez (q.d.D.g) en la entrega del galardón Alfonso Carro Zúñiga. San José:
Cátedra Teoría del Estado. Facultad de Derecho, Universidad de Costa Rica, junio.

Alfaro, José Miguel y Carlos Manuel Echeverría (1996). Procesos de integración centroamericana como región de paz, libertad, democracia y desarrollo. San José: Universidad para la Paz.

Asamblea Legislativa de la República de Costa Rica. Acta de la Sesión Plenaria n. ${ }^{\circ} 58$ (lunes 2 de setiembre de 2013). Cuarta Legislatura (del 1. ${ }^{\circ}$ de mayo de 2013 al 30 de abril de 2014). Segundo Período de Sesiones Ordinarias (del 1. ${ }^{\circ}$ de setiembre al 30 de noviembre de 2013). San José: Departamento de Servicios Parlamentarios. Área de Actas, Sonido y Grabación.

Asamblea Representativa Universitaria de la Universidad Estatal a Distancia. Acta n. ${ }^{\circ} 081-2010,5$ de marzo de 2010 .

Consejo Universitario de la Universidad Estatal a DisTANCIA. Sesión 1897-2007, correspondiente al 7 de diciembre del 2007. San José.

Consejo Universitario de la Universidad Estatal a DisTANCIA. Sesión 1987-2009, correspondiente al 16 julio 2009. San José.

Cuevas, Rafael y otros (2004). Integración en Centroamérica: cultural, social, política y económica. Heredia: Editorial de la Universidad Nacional.

Zeledón, Cristina (comp.) (2011). Carazo en el corazón del pueblo. Cartago: Editorial Tecnológica de Costa Rica. 
